\title{
Ejection of Glycine Molecules Adsorbed on a Water Ice Surface by Swift-heavy Ion Irradiation
}

\author{
Christian Anders ${ }^{1}$, Eduardo M. Bringa ${ }^{2}$, and Herbert M. Urbassek ${ }^{1}$ (1) \\ ${ }^{1}$ Physics Department and Research Center OPTIMAS, University Kaiserslautern, Erwin-Schrödinger-Straße, D-67663 Kaiserslautern, Germany \\ urbassek@rhrk.uni-kl.de \\ ${ }^{2}$ CONICET and Facultad de Ingenería, Universidad de Mendoza, Mendoza 5500, Argentina \\ Received 2019 October 1; revised 2020 January 10; accepted 2020 January 21; published 2020 February 28
}

\begin{abstract}
Organic molecules may be adsorbed on the ice surfaces of comets or moons. We study the desorption process induced by swift-heavy ion irradiation using a molecular dynamics simulation. Focusing on the amino acid glycine adsorbed on water ice as a prototypical example, we model a $2 \mathrm{MeV}$ sulfur ion impact as it might be typical of magnetospheric ion impact on the surface of Europa. We find that molecules are ejected intact within a radius of up to $25 \AA$ around the ion impact point. Within a core region of around $10 \AA$, glycine molecules are destroyed and mainly fragments are emitted. Prominent fragments produced are cyanide $\mathrm{CN}^{-}$, carbon monoxide $\mathrm{CO}$, cyanate $\mathrm{OCN}^{-}$, and carbon dioxide $\mathrm{CO}_{2}$, in agreement with experimental studies. In addition, radiolysis of water ice generates the radicals $\mathrm{H}^{+}, \mathrm{H}_{3} \mathrm{O}^{+}$, and $\mathrm{HO}^{-}$as well as the gases $\mathrm{H}_{2}, \mathrm{O}_{2}$, and some $\mathrm{H}_{2} \mathrm{O}_{2}$. While the smaller fragments easily obtain velocities above $2 \mathrm{~km} \mathrm{~s}^{-1}$ - the escape velocity from Europa-most ejected glycine molecules obtain smaller velocities and will thus not leave the moon permanently. Our results thus provide a detailed example that shows to what extent intact emission of organic molecules from Europa's surface by ion irradiation is possible and may be used for modeling the height distribution of ejecta in Europa's exosphere.
\end{abstract}

Unified Astronomy Thesaurus concepts: Computational methods (1965); Galilean satellites (627); Astrochemistry (75)

\section{Introduction}

Ice surfaces are ubiquitous in the outer solar system (Gudipati \& Castillo-Rogez 2013), where many moons are covered by an ice shield and also comets consist mainly of ice. Also in the inner solar system, ice is found in places not lit by the Sun, such as on Earth's moon, or in the asteroid belt on Ceres. The composition of these ices is complex; besides water, many more substances such as $\mathrm{CO}_{2}, \mathrm{NH}_{3}$, or $\mathrm{CH}_{3} \mathrm{OH}$ (Martins et al. 2013) are contained in the ices. In addition, recent results on the composition of the comet 67P/Churyumov-Gerasimenko found organic molecules (Altwegg et al. 2016). The amino acid glycine was identified as the most prominent molecule.

These materials are constantly irradiated by solar-wind particles, by magnetospheric ions and also by cosmic radiation (Johnson 1990). As a consequence, the ices are chemically transformed, and material may also be sputter-ejected from the surface. In addition, future space missions may use ion irradiation to artificially desorb material from the surface in order to analyze it (Meyer et al. 2017; Teolis et al. 2017; Johnson \& Sundqvist 2018).

Jupiter's ice moon Europa is the objective of the coming JUICE mission (Grasset et al. 2013). It is a fascinating moon since below its ice surface, a subsurface ocean is expected (Pappalardo et al. 2009). While organic material has not yet been found on Europa's surface (Carlson et al. 2009), one may speculate about its presence, either originating from the ocean from which geological processes may have brought it to the ice surface or having been deposited from comets impacting the surface. It has even been considered to what extent heavy-ion irradiation might eject molecules from the surface, which could be used as a probe of the ice (or ocean) composition (Johnson \& Sundqvist 1992; Johnson et al. 1998; Johnson \& Sundqvist 2018).

High temperatures and pressures - such as those created during ion irradiation-may induce reactions among the molecules present in the surface material. Extremely high temperatures and pressures may also occur during shock loading (Goldman \& Tamblyn 2013; Martins et al. 2013; Koziol \& Goldman 2015) of an ice sample-for instance induced by asteroid impact-or during cavitation bubble collapse (Kalson et al. 2017; Wu \& Adnan 2017), and the resulting reactions in prebiotic ice mixtures have been described by using an atomistic simulation.

In this paper, we use a molecular dynamics simulation to investigate the effect of ion irradiation on glycine molecules adsorbed on the surface of water ice. Besides the fragmentation of the molecules, we focus on the ion-induced emission of material from the surface. In particular, the possibility of intact emission of glycine molecules is studied.

\section{Method}

We prepare a cuboid amorphous water ice target using PACKMOL (Martínez et al. 2009). It has extensions of $204 \AA \times$ $104 \AA$ on the surface and a depth of $212 \AA$. On the top surface we position glycine (Gly) molecules, $\mathrm{H}_{2} \mathrm{~N}-\mathrm{CH}_{2}-\mathrm{COOH}$, see Figure 1 . Rather than performing a multitude of simulations in which only a single isolated glycine molecule is situated on the surface, we position 1000 of them on the surface with an average distance of $2 \AA$.

The system is relaxed in two steps, first a damped molecular dynamics simulation under periodic boundary conditions is used, then an open top surface is created. The system is equilibrated for $18 \mathrm{ps}$ at a temperature of $101 \mathrm{~K}$, corresponding to the surface temperature of Europa. The amorphous ice has a mass density of $0.957 \mathrm{~g} \mathrm{~cm}^{-3}$ - corresponding to a molecular number density of $32 \mathrm{~nm}^{-3}$-in agreement with experiment (Tachibana et al. 2017).

We use a REAX potential (van Duin et al. 2001; Senftle et al. 2016) to model interatomic interactions, since it allows us 


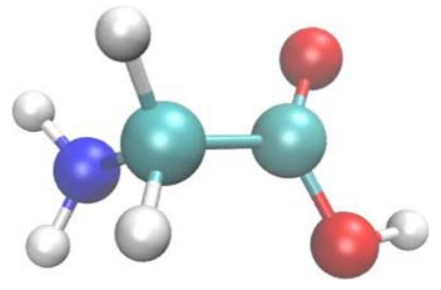

Figure 1. Glycine molecule visualized by VMD (Humphrey et al. 1996).

to consider molecule dissociations and reactions to be included in the molecular dynamics simulation; the version created by Monti et al. (2013) is used because it has been specially optimized for simulations involving glycine. Toward high energies, we spline the two-body part of these potentials to the Ziegler-Biersack-Littmark (ZBL) potential (Ziegler et al. 1985), in order to allow for realistic atom-collision physics at high energies. The spline starts to modify the potentials above around $10 \mathrm{eV}$; this will only affect the projectile-target interaction and particles in the Maxwell tails of the track. Details of the spline are given by Anders \& Urbassek (2013).

The system is irradiated by a $2 \mathrm{MeV} \mathrm{S}$ ion at an angle of $45^{\circ}$ to the surface normal such that the azimuth direction is aligned with the long edge length of the simulation volume. At the sides and the bottom of the simulation volume, a Berendsen thermostat (Berendsen et al. 1984) is used in a $10 \AA$ wide zone to keep the temperature at $101 \mathrm{~K}$. Atoms leaving the simulation volume toward the sides or the bottom are stopped, similar to previous simulations (Anders \& Urbassek 2019a, 2019b). The top surface is left free. The projectile ion suffers nuclear collisions with target atoms according to the ZBL potential (Ziegler et al. 1985). In addition, it loses energy by electronic stopping; according to Ziegler (2000), the electronic stopping power amounts to $89 \mathrm{eV}^{-1}$. We note that for a $2 \mathrm{MeV} \mathrm{S}$ ion in ice, only around $1.5 \%$ of the energy loss go into nuclear stopping while the dominant part goes into electronic stopping (Ziegler 2000; Anders \& Urbassek 2019a). This electronic stopping leads to an energy transfer from the ion to the target that is in reality mediated by the excitation and ionization of target electrons. However, as it is typical for ion-track simulations (Fenyö et al. 1990; Urbassek et al. 1994; Pakarinen et al. 2009; Papaléo et al. 2015), we model energy transfer via a thermal-track model, as detailed in previous work (Mainitz et al. 2016, 2017; Anders \& Urbassek 2019a, 2019b). In short, the atoms in a cylindrical region of radius $R$ around the ion trajectory are given a thermal energy such that the total transferred energy per ion path length equals the stopping power. In agreement with previous work (Mainitz et al. 2016, 2017; Anders \& Urbassek 2019a, 2019b), we set $R=5 \AA$, and hence the initial track temperature amounts to $56,500 \mathrm{~K}$; this high temperature is needed to obtain the dissociations and ensuing reactions that are caused in reality by the electron excitation and ionization processes induced by the swift-heavy ion. We note that in reality not the entire energy deposited in electronic excitation will be transferred as thermal energy to the molecules (Johnson \& Brown 1982); this will result in a smaller number of chemical transformations and a lower ejection yield, while the qualitative features of the processes remain unchanged, as we verified in our previous simulations of irradiation of ice targets (Mainitz et al. 2016, 2017). The simulation runs for $32.1 \mathrm{ps}$.

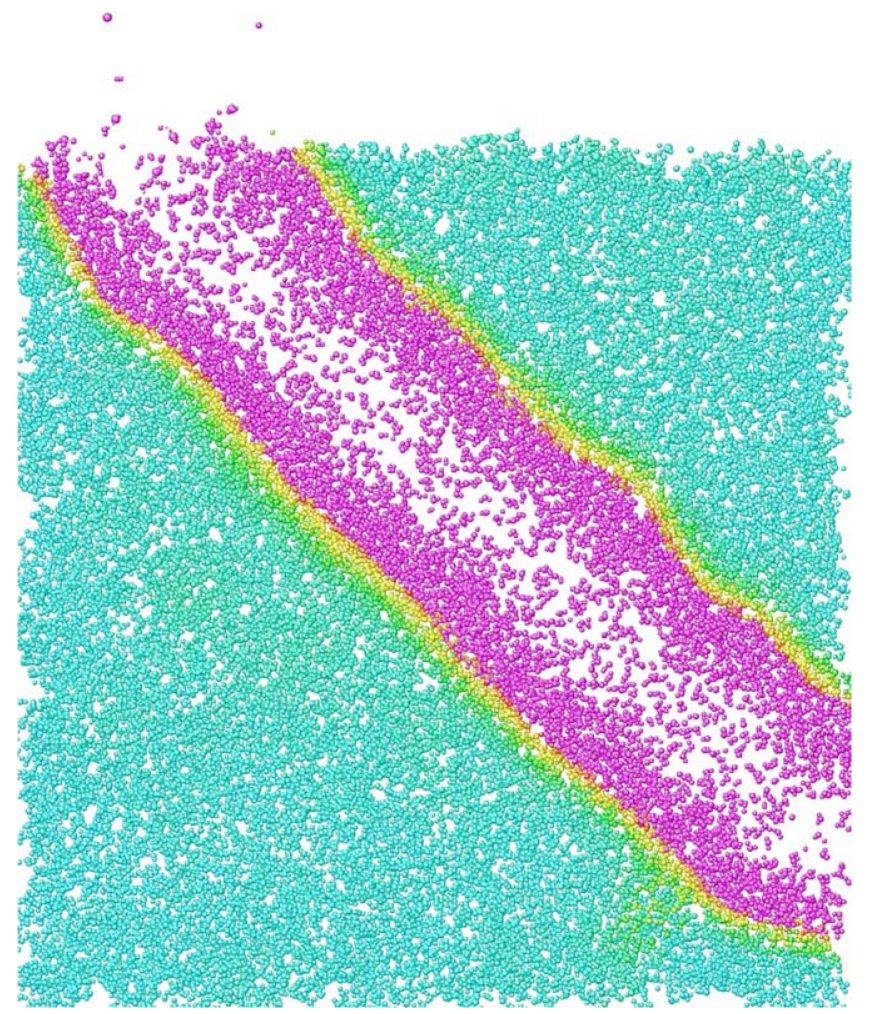

Figure 2. Snapshot of the ion track at a time of 0.5 ps after track formation. Several ejecta are already seen above the track. The figure shows a slab of $1.4 \mathrm{~nm}$ thickness, width of $19.2 \mathrm{~nm}$, and height of $24 \mathrm{~nm}$. The color represents the local temperature varying between cyan $(101 \mathrm{~K}$, corresponding to the surface temperature of Europa) and purple $(>373 \mathrm{~K})$.

The size of the simulation volume (see Figure 2) has been set large enough to include the near-surface part of the track entirely and also the region relevant for particle ejectionextending around $25 \AA$ from the ion track axis, see Figures 5, 6, and 9(a) below. Since the effects relevant for chemical transformation and for ejection occur mainly in the ion track region, we are therefore confident that the irradiation effects responsible for chemical transformation and molecule ejection are faithfully included. The damping of atoms in the lateral and bottom boundaries might somewhat change the material physics - such as the final density - in the track at late times; we do not investigate these effects, since they are irrelevant to the emission phenomena of interest here. We note that our simulation volume is of comparable size as that used for modeling ion-track phenomena in other systems (Fenyö et al. 1990; Urbassek et al. 1994; Pakarinen et al. 2009; Papaléo et al. 2015).

Simulations are performed using the "Large-scale Atomic/ Molecular Massively Parallel Simulator" (LAMMPS) opensource software (Plimpton 1995). Figure 2 illustrates the ion track in the target at a time of 0.5 ps after $\mathrm{S}$ ion impact.

\section{Results}

After equilibration at $101 \mathrm{~K}$, the chemical composition of the system-which originally consists of only water and glycine molecules - has slightly changed, see Table 1. Glycine is zwitter-ionic and easily accepts or donates a proton (Vollhardt \& Schore 2014); this behavior is reproduced by the interatomic interaction potential used in our simulation 
Table 1

Target Composition Immediately before Track Formation

\begin{tabular}{lccc}
\hline \hline Molecule & Mass (amu) & $N$ & Comment \\
\hline $\mathrm{C}_{2} \mathrm{H}_{4} \mathrm{NO}_{2}$ & 74 & 172 & Gly-H \\
$\mathrm{C}_{2} \mathrm{H}_{5} \mathrm{NO}_{2}$ & 75 & 775 & Gly \\
$\mathrm{C}_{2} \mathrm{H}_{6} \mathrm{NO}_{2}$ & 76 & 53 & Gly+H \\
\hline $\mathrm{H}^{+}$ & 1 & 29 & \\
$\mathrm{H}_{2} \mathrm{O}$ & 18 & 106274 & \\
$\mathrm{H}_{3} \mathrm{O}^{+}$ & 19 & 90 & \\
\hline
\end{tabular}

Note. $N$ : absolute number of molecules used in the simulation.

(Rahaman et al. 2011). Here and in the following we abbreviate deprotonated glycine by "Gly-H" and protonated glycine by "Gly+H." Also some dissociations in water occur, forming around 1 per mille $\mathrm{H}^{+}$and $\mathrm{H}_{3} \mathrm{O}^{+}$.

\subsection{Chemical Transformations}

Figure 3 shows the time evolution of the number of molecules produced by the impact. As the curves saturate toward the end of the simulation, we are confident that all impact-related dissociations and chemical reactions have been captured in our simulation and that a large enough simulation time has been chosen. We mention that at the end of the simulation, 32.1 ps after track formation, the average temperature in the track is $195 \mathrm{~K}$, while fluctuations may reach up to $373 \mathrm{~K}$. Note that water molecules are destroyed by the impact and radiolysis products- $\mathrm{H}^{+}, \mathrm{OH}^{-}$, and $\mathrm{H}_{3} \mathrm{O}^{+}$- were created. However, due to ongoing reactions, the loss in water molecules is partly healed during the course of the simulation.

Further reactions between the radiolysis products lead to the generation of $\mathrm{H}_{2}$ and $\mathrm{O}_{2}$. This occurs almost instantaneously, within $1 \mathrm{ps}$ of the ion impact. The generation of these molecules is in agreement with laboratory experiments on the radiolysis of water ice (Bar-Nun et al. 1985; Baragiola et al. 2005).

A complete list of the species generated by the impact is assembled in Table 2. The species originating from water fragmentation are listed in the lowest rows, as they contain only $\mathrm{H}$ and $\mathrm{O}$. Dissociation products of glycine, as well as reaction products of these fragments, contain $\mathrm{N}$ and $\mathrm{C}$ and are found in the upper rows. Not unexpectedly, a large number of glycine molecules (189) were destroyed. On the other hand, 163 Gly have gained or lost one proton; we will denote these molecules as Gly $\pm \mathrm{H}$. In view of the zwitter-ionic nature of glycine, Gly $\pm \mathrm{H}$ cannot be considered as an essentially new product molecule. The other products are mainly fragments, and their atomic mass is smaller than that of glycine. The only exception is made by $\mathrm{C}_{2} \mathrm{H}_{7} \mathrm{NO}_{2}$, which represents a doubly protonated glycine.

We note that dissociations by direct projectile impacts are almost negligible compared to the large number of bond breakings occurring in the thermal spike. From our simulation, we determine that only $E_{n}=0.4 \mathrm{keV}$ of the projectile energy goes into direct nuclear collisions with the impinging $\mathrm{S}$ ion. The number of dissociations caused hereby can be estimated as $E_{n} /(8 D)$, where $D$ is a dissociation energy (Balaji et al. 1995; Anders \& Urbassek 2017). With a representative value of $D=5 \mathrm{eV}$, we conclude that only 10 dissociations are caused by nuclear collisions.

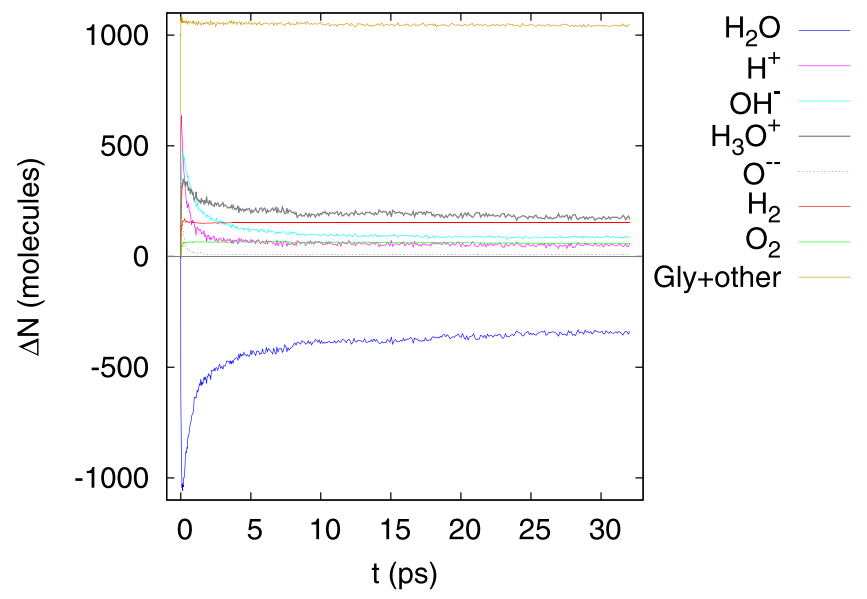

Figure 3. Temporal evolution of the change in the number of molecules, $\Delta N$, after track formation at time $t=0$. The figure focuses on the changes in the radiolysis products of water. Changes in glycine and its fragments occur almost instantaneously and are summarized in the curve "Gly+other."

In particular, we did not observe the formation of di-glycine, i.e., a peptide, which would necessitate the formation of a peptide bond (-CO-NH-). The formation of peptide bonds would be interesting, as it would indicate the possible formation of prebiotic molecules in swift-ion tracks. The lack of peptide-bond formation is, however, understandable, since the high temperatures (or pressures) necessary for forming such a bond last only for a very short time in the aftermath of the swift-ion impact. Shock-induced glycine polymerization was observed for simulations where pressures higher than $35 \mathrm{GPa}$ (and the resulting high temperatures) were held during $100 \mathrm{ps}$ (Martins et al. 2013; Koziol \& Goldman 2015) in contrast to the pressure history of the ion track lasting only a fraction of a ps and reaching only $100 \mathrm{MPa}$ peak pressure. In fact, temperatures above $1000 \mathrm{~K}$ and pressures above a few tens of $\mathrm{MPa}$ last in the ion track only around $5 \mathrm{ps}$. In addition, pressures in the track center soon become tensile, since it becomes underdense as molecules have been pushed radially away. Furthermore, in a series of studies, Goldman and coworkers found that the energy barrier for glycine dimerization is around $20 \mathrm{kcal} \mathrm{mol}^{-1}$ or roughly $0.9 \mathrm{eV}$ (Kroonblawd et al. 2018; Lindsey et al. 2019). However, in our study, glycine molecules left in the surface reach kinetic energies of around $0.5 \mathrm{eV}$ at maximum. This energy is of the order of the cohesive energy of water- $0.52 \mathrm{eV}$ (Johnson 1990). Only ejected glycines may reach higher energies, above $1 \mathrm{eV}$ (see Section 3.2); however, these are emitted immediately after track formation and hence have no time for bond formation. In conclusion, in spite of the high initial temperatures and pressures formed in the ion track, the thermodynamic state soon (within $5 \mathrm{ps}$ ) becomes too close to equilibrium to allow for peptide bond formation.

Disregarding the glycines which only lost or captured hydrogen atom, 973 of the 1000 original glycine molecules, i.e., $97.3 \%$, remain intact, and 27 are fragmented.

Figure 4(b) shows the generation of new species in the form of a simulated mass spectrum and compares it with the original spectrum before impact (Figure 4(a)).

Besides the generation of the organic glycine fragments, prominent products are $\mathrm{H}_{2} \mathrm{O}_{2}, \mathrm{CO}_{2}, \mathrm{NH}_{3}$, and $\mathrm{H}_{2} \mathrm{~S}$.

Some molecules are ejected after the ion impact. These are listed in Table 3, while their mass spectrum is shown in 
Table 2

Number of Molecules $N$ at the End of the Simulation, 32.1 ps after Track Formation

\begin{tabular}{|c|c|c|c|}
\hline Molecule & Mass (amu) & $N$ & Comment \\
\hline $\mathrm{C}$ & 12 & 2 & \\
\hline $\mathrm{CO}$ & 28 & 3 & Exp \\
\hline $\mathrm{CO}_{2}$ & 44 & 12 & Exp \\
\hline $\mathrm{CN}$ & 26 & 3 & Exp \\
\hline $\mathrm{CNO}$ & 42 & 2 & Exp \\
\hline $\mathrm{CHO}$ & 29 & 3 & \\
\hline $\mathrm{CHO}_{2}$ & 45 & 2 & \\
\hline $\mathrm{CH}_{3}$ & 15 & 1 & \\
\hline $\mathrm{CH}_{3} \mathrm{~N}$ & 29 & 2 & \\
\hline $\mathrm{CH}_{4} \mathrm{~N}$ & 30 & 2 & \\
\hline $\mathrm{C}_{2} \mathrm{H}_{2} \mathrm{O}$ & 42 & 2 & \\
\hline $\mathrm{C}_{2} \mathrm{H}_{3} \mathrm{O}_{2}$ & 59 & 2 & \\
\hline $\mathrm{C}_{2} \mathrm{H}_{3} \mathrm{O}_{3}$ & 75 & 1 & \\
\hline $\mathrm{C}_{2} \mathrm{H}_{3} \mathrm{NO}$ & 57 & 2 & \\
\hline $\mathrm{C}_{2} \mathrm{H}_{4} \mathrm{O}_{2}$ & 60 & 1 & \\
\hline $\mathrm{C}_{2} \mathrm{H}_{4} \mathrm{NO}_{2}$ & 74 & 266 & Gly-H: $\Delta N=+94$ \\
\hline $\mathrm{C}_{2} \mathrm{H}_{5} \mathrm{NO}$ & 59 & 1 & \\
\hline $\mathrm{C}_{2} \mathrm{H}_{5} \mathrm{NO}_{2}$ & 75 & 586 & Gly: $\Delta N=-189$ \\
\hline $\mathrm{C}_{2} \mathrm{H}_{6} \mathrm{NO}_{2}$ & 76 & 122 & Gly $+\mathrm{H}: \Delta N=+69$ \\
\hline $\mathrm{C}_{2} \mathrm{H}_{7} \mathrm{NO}_{2}$ & 77 & 1 & \\
\hline $\mathrm{H}_{2} \mathrm{~S}$ & 34 & 1 & \\
\hline $\mathrm{N}$ & 14 & 1 & \\
\hline $\mathrm{NH}$ & 15 & 1 & \\
\hline $\mathrm{NH}_{2}$ & 16 & 1 & \\
\hline $\mathrm{NH}_{3}$ & 17 & 10 & \\
\hline $\mathrm{H}$ & 1 & 89 & $\Delta N=+60$ \\
\hline $\mathrm{H}_{2}$ & 2 & 152 & \\
\hline $\mathrm{O}$ & 16 & 9 & \\
\hline $\mathrm{OH}$ & 17 & 84 & \\
\hline $\mathrm{H}_{2} \mathrm{O}$ & 18 & 105936 & $\Delta N=-338$ \\
\hline $\mathrm{H}_{3} \mathrm{O}$ & 19 & 167 & $\Delta N=+77$ \\
\hline $\mathrm{O}_{2}$ & 32 & 60 & \\
\hline $\mathrm{O}_{2} \mathrm{H}$ & 33 & 1 & \\
\hline $\mathrm{H}_{2} \mathrm{O}_{2}$ & 34 & 10 & \\
\hline $\mathrm{H}_{3} \mathrm{O}_{2}$ & 35 & 13 & \\
\hline
\end{tabular}

Note. The table is ordered in groups containing carbon, sulfur, nitrogen, hydrogen, and oxygen. Molecules found in experimental work of glycine irradiation (Portugal et al. 2014) are marked by "Exp." Changes to original abundances, Table 1, are denoted by $\Delta N$.

Figure 4(c). The majority of the emitted species are water molecules, followed by bare protons. Volatile gases, in particular $\mathrm{H}_{2}$, but also $\mathrm{NH}_{3}$, also easily desorb. However, also 15 Gly $\pm \mathrm{H}$ are desorbed. While these constitute only a minority $(1.5 \%)$ of the glycines present in the sample, it is reassuring to observe the intact ejection of glycine molecules from the target surface, in agreement with previous experiments that showed the possibility of intact ejection of biomolecules by swift-heavy-ion irradiation (Hedin et al. 1987; Ens et al. 1989; Johnson \& Sundqvist 1992).

We note that while $\mathrm{O}_{2}$ has been produced in our simulations, no $\mathrm{O}_{2}$ molecules are ejected. This might appear astonishing since it is known that Europa has a tenuous atmosphere containing $\mathrm{O}_{2}$, and its existence was ascribed to irradiation effects (Hall et al. 1995; Shematovich \& Johnson 2001; Johnson et al. 2004, 2009; Teolis et al. 2017). However, it is known that the sputtering of $\mathrm{O}_{2}$ from water ice exhibits a strong fluence effect in that at low fluences-such as in our simulation

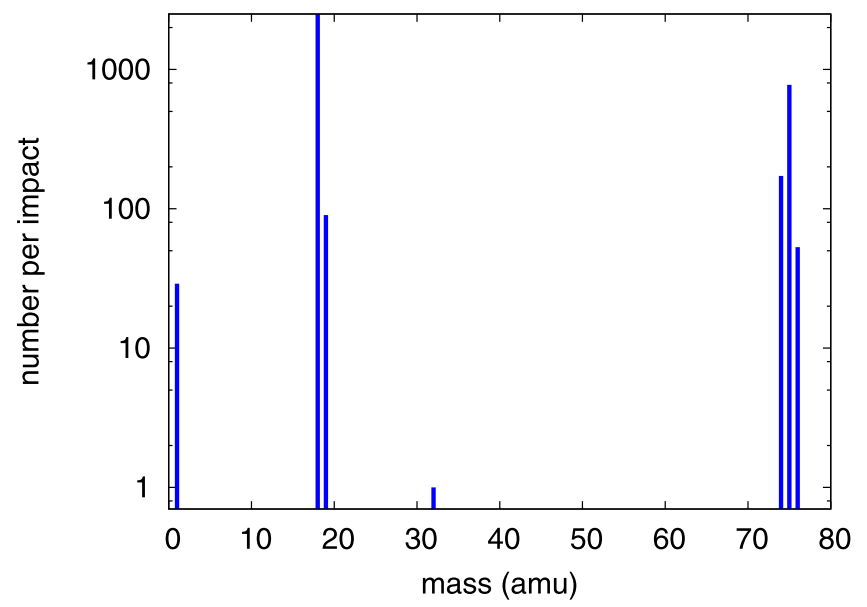

(a)

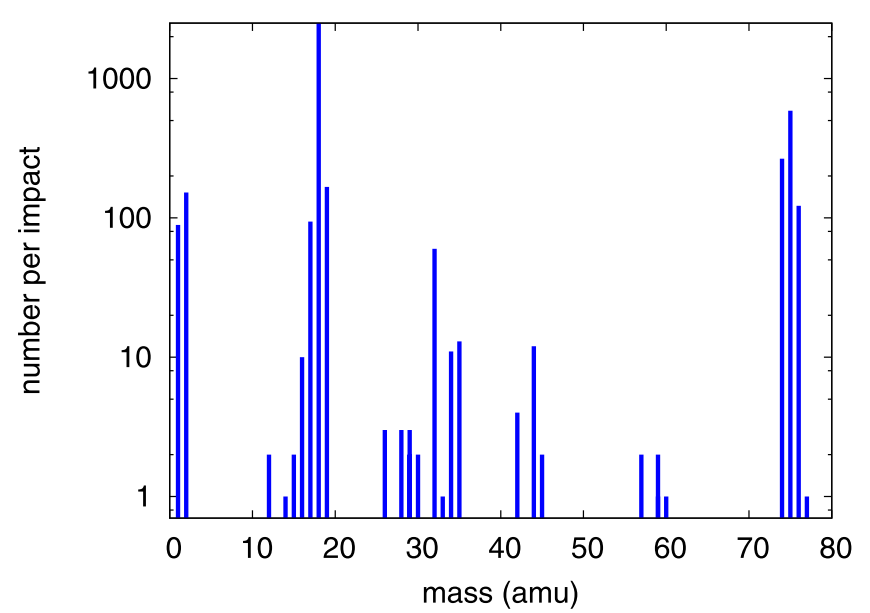

(b)

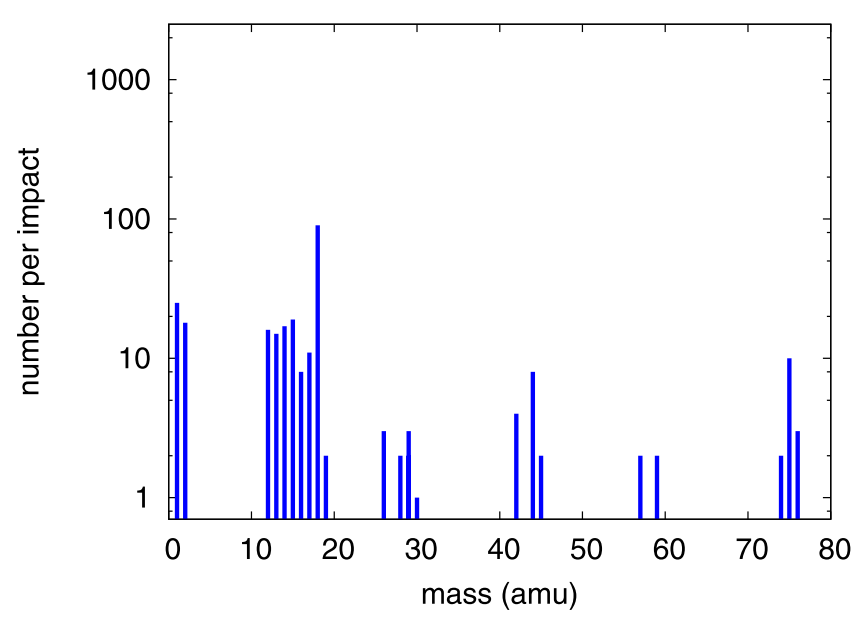

(c)

Figure 4. Simulated mass spectrum showing target composition (a) immediately before track formation, and (b) at the end of the simulation, 32.1 ps. (c) shows the mass spectrum of ejecta.

- emission is negligible but increases during ongoing irradiation to a steady state value (Teolis et al. 2005). The fluence effect has been assigned to the build-up of trapped $\mathrm{O}_{2}$ near the 
Table 3

Number $N$ of Ejected Atoms and Molecules

\begin{tabular}{lcc}
\hline \hline Sum Formula & Mass (amu) & $N$ \\
\hline $\mathrm{C}$ & 12 & 2 \\
$\mathrm{CO}$ & 28 & 2 \\
$\mathrm{CO}_{2}$ & 44 & 8 \\
$\mathrm{CN}$ & 26 & 3 \\
$\mathrm{CNO}$ & 42 & 2 \\
$\mathrm{CHO}$ & 29 & 3 \\
$\mathrm{CHO}_{2}$ & 45 & 2 \\
$\mathrm{CH}_{3}$ & 15 & 1 \\
$\mathrm{CH}_{3} \mathrm{~N}$ & 29 & 2 \\
$\mathrm{CH}_{4} \mathrm{~N}$ & 30 & 1 \\
$\mathrm{C}_{2} \mathrm{H}_{2} \mathrm{O}$ & 42 & 2 \\
$\mathrm{C}_{2} \mathrm{H}_{3} \mathrm{O}_{2}$ & 59 & 2 \\
$\mathrm{C}_{2} \mathrm{H}_{3} \mathrm{NO}_{\mathrm{C}_{2} \mathrm{H}_{4} \mathrm{NO}_{2}}$ & 27 \\
$\mathrm{C}_{2} \mathrm{H}_{5} \mathrm{NO}_{2}$ & 74 & 2 \\
$\mathrm{C}_{2} \mathrm{H}_{6} \mathrm{NO}_{2}$ & 75 & 10 \\
\hline $\mathrm{N}$ & 76 & 3 \\
$\mathrm{NH}$ & 14 & 1 \\
$\mathrm{NH}_{2}$ & 15 & 1 \\
$\mathrm{NH}_{3}$ & 16 & 1 \\
\hline $\mathrm{H}$ & 17 & 7 \\
$\mathrm{H}_{2}$ & 1 & 25 \\
$\mathrm{O}$ & 2 & 18 \\
$\mathrm{OH}$ & 16 & 7 \\
$\mathrm{H}_{2} \mathrm{O}$ & 17 & 2 \\
$\mathrm{H}_{3} \mathrm{O}$ & 18 & \\
\hline & 19 & 2 \\
\hline & & 2 \\
\hline
\end{tabular}

Note. The table is ordered in groups containing carbon, nitrogen, hydrogen, and oxygen.

surface and the subsequent release under prolonged irradiation (Teolis et al. 2009).

The radiolysis products of glycine include cyanide $\mathrm{CN}^{-}$, carbon monoxide $\mathrm{CO}$, cyanate $\mathrm{OCN}^{-}$, and carbon dioxide $\mathrm{CO}_{2}$, but also $\mathrm{H}_{2} \mathrm{O}$, as a detailed study of the origin of the newly formed water molecules reveals. We note that an experimental study by Portugal et al. (2014) on glycine irradiation showed exactly these same products; this fact nicely confirms our results.

In total, summing over both glycine and water molecules, we find a number of $N_{\text {diss }}=531$ dissociations and $N_{\text {prod }}=675$ new product molecules formed in our sample as a consequence of the impact. We can compare this number to other studies by referring to the total energy deposited by the ion in the simulation volume, $E_{\mathrm{dep}}$. With a stopping power of $89 \mathrm{eV} \AA^{-1}$ and a track length of $239 \AA$ we have $E_{\text {dep }}=21.2 \mathrm{keV}$. As shown in previous work (Anders \& Urbassek 2017, 2019a), the number of dissociations and product molecules is proportional to $E_{\mathrm{dep}}$. We therefore obtain proportionality constants of $a=N_{\text {diss }} / E_{\text {dep }}=25 \mathrm{keV}^{-1}$ and $b=N_{\text {prod }} / E_{\text {dep }}=32 \mathrm{keV}^{-1}$. This is similar to the values found for $\mathrm{keV}$ impacts (Anders \& Urbassek 2017), but smaller (by around 50\%) than the values for impacts into an ice mixture consisting of $\mathrm{H}_{2} \mathrm{O}, \mathrm{CO}_{2}, \mathrm{NH}_{3}$, and $\mathrm{CH}_{3} \mathrm{OH}$ (Anders \& Urbassek 2019a). We presume that this reduction in dissociation and reaction efficiency is caused by the fact that the number of reaction channels in a pure water target is considerably smaller than in the ice mixture.

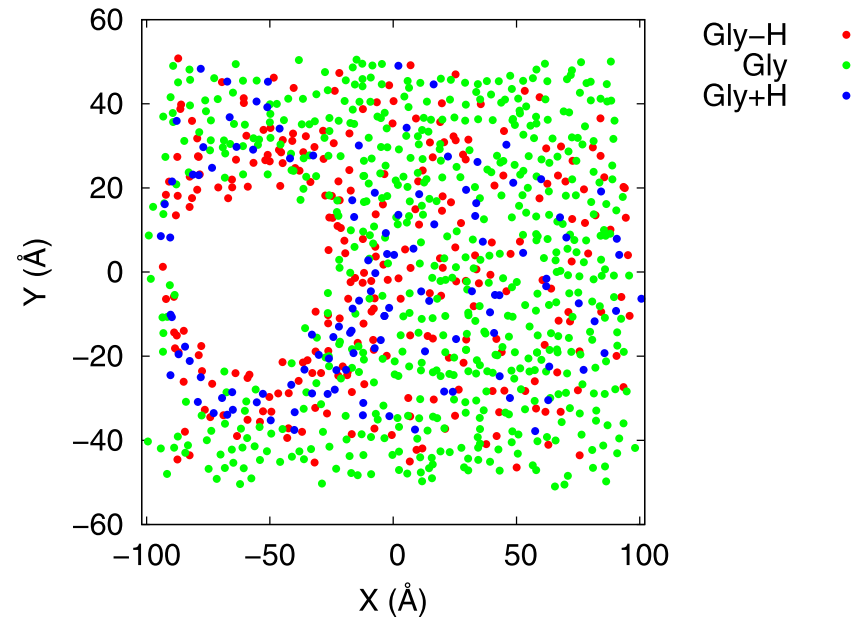

Figure 5. Top view of glycine positions on the surface at the end of the simulation. Note that the $x$ - and $y$-axes use different scales.

\subsection{Emission from the Surface}

Initially, the glycine molecules covered the ice surface homogeneously. After the impact, the region around the ion incidence point on the surface is cleared from glycine, see Figure 5. While some molecules were ejected, the other glycines were moved aside and formed a sort of rim around the impact region, where the glycine concentration is enhanced. In addition, in this rim, the majority of Gly $\pm \mathrm{H}$ molecules are found, while initially the glycine molecules dominated. This feature shows the enhanced reaction probability in the vicinity of the impact point.

The original positions of ejected glycines are also indicated in Figure 6, where the positioning of the ejected molecules with respect to the ion track can be seen more clearly. Evidently, more molecules to the front of the ion track were emitted than in its back. Figure 6(b) also includes the original positions of the fragments formed. Fragments containing $\mathrm{N}$ or $\mathrm{C}$ stem from the surface layer of glycines covering the ice; this is evident as they must originate from glycine molecules. However, fragments of water molecules also originate from greater depths-exceeding $50 \AA$; here, however, only fragments produced in the immediate vicinity of the ion track could be emitted. Such an emission is possible, since the ion track becomes underdense after the passage of the ion due to the high temperature and pressures existing there; this makes fragment emission from such depths possible.

The total mass of the ejecta amounts to $4379 \mathrm{amu}$; this corresponds to the mass of 58 glycine molecules.

The radial distance to the track, from which glycine molecules and fragments were ejected, is shown in Figure 7. Molecules and fragments are emitted up to a maximum distance of around $r=(25 \pm 5) \AA$. This translates to a desorption cross section of $\pi r^{2}=(19.6 \pm 7.9) \mathrm{nm}^{2}$. Intact emitted glycine molecules, however, rarely originate from the inner part of the track. Within a core region of around $10 \AA$, glycine molecules are destroyed and mainly fragments are emitted; within $2 \AA$ from the track all glycines fragmented. In these inner regions, however, fragment emission of glycine is active, as well as emission of water fragments.

The velocities of ejecta are relevant, since they may be large enough to surpass the gravitational attraction of the parent body. Taking the Jovian moon Europa as an example, its 


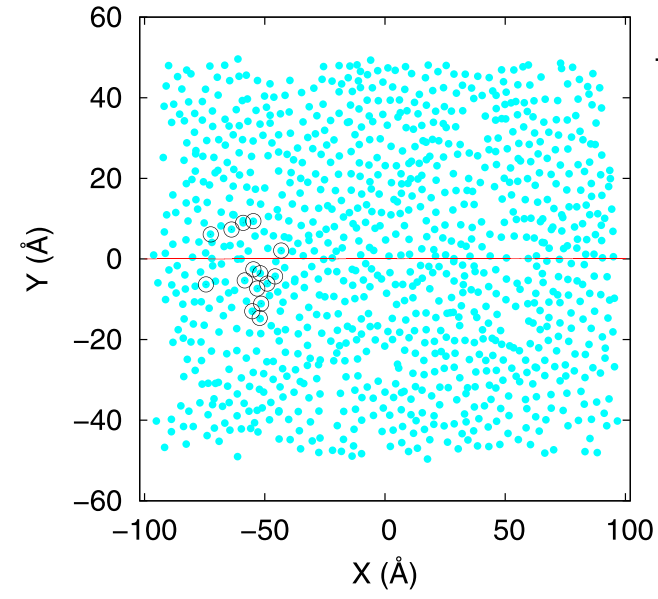

(a)

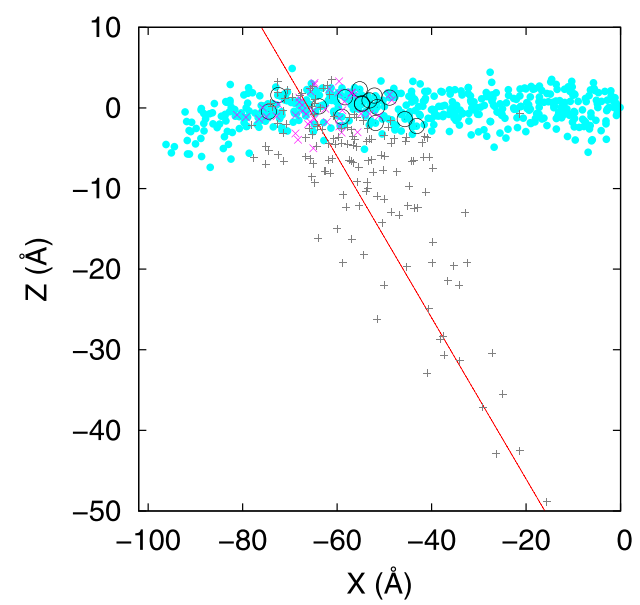

(b)

Figure 6. (a) Top view and (b) side view of initial glycine positions on the surface. Projection of ion track and initial positions of ejected intact glycine molecules as well as fragments are highlighted. Fragments containing $\mathrm{N}$ or $\mathrm{C}$ must originate from glycine; other fragments-denoted as "fragm $\mathrm{O}, \mathrm{H}$ "mostly originate from dissociated water.

escape velocity amounts to $v_{\text {escape }}=2.03 \mathrm{~km} \mathrm{~s}^{-1}$. Molecules with smaller velocity will be deposited elsewhere on the surface of the parent body.

The maximum center-of-mass velocity of an ejected molecule is taken as the molecule velocity $v$; from this its translational energy $E_{\text {trans }}$ is calculated. These data also allow us to determine the direction of motion of the ejecta.

Figures 8(a) and (b) show the velocity and energy distribution of ejecta. Most glycines have small velocity, below $v_{\text {escape }}$-energies below $3 \mathrm{eV}$-and will therefore not escape from the moon; only $20 \%$ (three molecules) have attained velocities above $v_{\text {escape }}$. However, the situation is different for the fragments; they have mostly velocities that let them escape, and their energies extend beyond $10 \mathrm{eV}$. In fact, sputter-induced molecule emission from the icy moons is a well-known phenomenon that has been repeatedly reported in the past (Johnson 1990; Paranicas et al. 2009; Cassidy et al. 2013); for Europa, the contribution of sputtering to a tenuous atmosphere has also been shown (Shematovich \& Johnson 2001; Johnson et al. 2009; Teolis et al. 2017; Vorburger \& Wurz 2018).

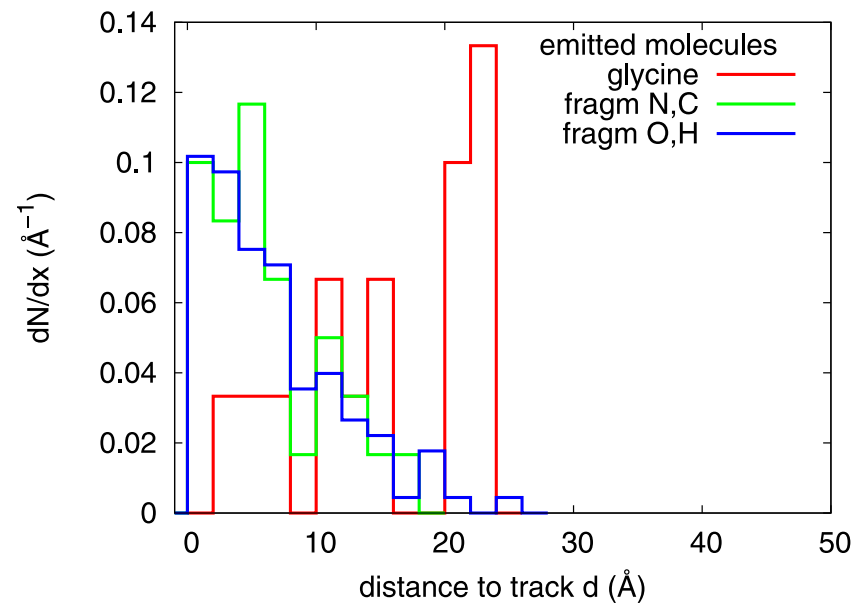

Figure 7. Distribution of emitted molecules as a function of the radial distance of their original location to the ion track. Fragments are denoted as in Figure 6. Distributions are normalized to area 1.

From our data, we may calculate the height, $h$, reached by the ejecta in their Kepler orbit above the surface of Europa; for this calculation only those ejecta that perform an elliptical trajectory have been included. Figure 8(c) shows the height distribution obtained, where the heights were normalized to Europa's radius, $R_{\mathrm{E}}$. The distribution can be fitted to a powerlaw dependence

$$
\frac{d N}{d h} \propto\left(\frac{h}{R_{\mathrm{E}}}\right)^{-n},
$$

with exponent $n=1.16$, both for glycine and for other ejecta. We note that from theoretical considerations, a height distribution $\propto\left[1+z / h_{c}\right]^{-1.5}$ with a scale height, $h_{c}$, depending on the ejected molecule was calculated (Johnson et al. 1982; Johnson 1990; Johnson \& Sundqvist 2018), corresponding to a power-law, Equation (1), with $n=1.5$. This latter distribution applies, however, to the density distribution in the vicinity of the moon, $z \ll R_{\mathrm{E}}$, rather than to the distribution of maximum heights reached that we evaluated.

We may correlate the emission velocities with the radial distance from the ion track from which the ejected molecules or fragments originate. Figure 9(a) shows this correlation. The glycines attain fairly constant velocities, which only little depend on their original distance to the ion track; as mentioned above, only three ejected glycines have high enough velocity to leave Europa. However, fragments may not only reach higher velocities, but their velocities are clearly correlated to the distance from the track; the farther from the track they originate the lower their velocity is. This correlation is caused by the decrease in pressure and in particular of temperature with distance from the track axis.

Figure 9(b) shows how the attained velocities are correlated with the mass of the ejected particle. Particles with higher mass tend to reach lower velocities than those with smaller mass. The intact glycine molecules are the heaviest ejecta and correspondingly have the lowest velocity.

Our data also allow us to determine the emission angles of the ejecta. Figure 10 shows the polar angles $\theta$ and the azimuthal angle $\varphi$ of the ejected molecules; here, $\theta$ gives the angle to the surface normal and $\varphi$ the azimuthal angle relative to the impact direction. Ejection of glycine occurs preferentially in the 


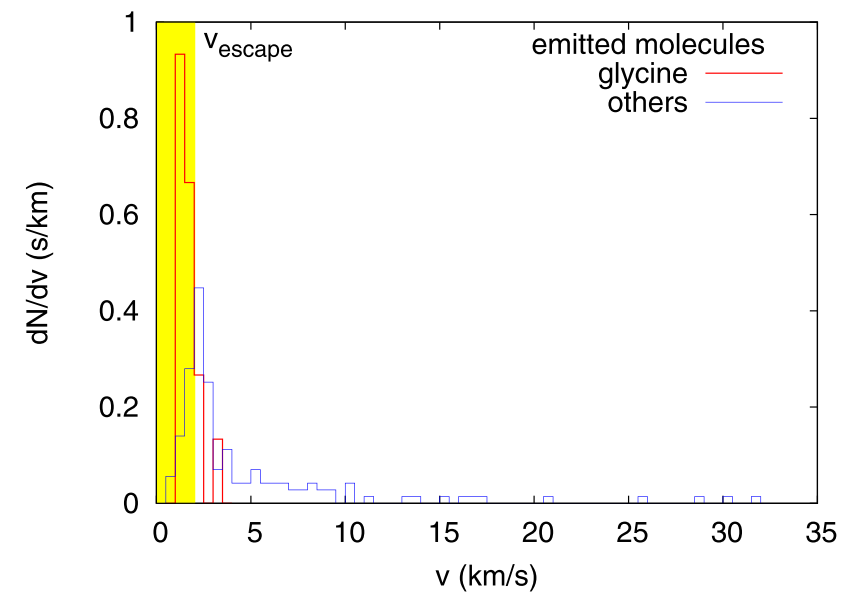

(a)

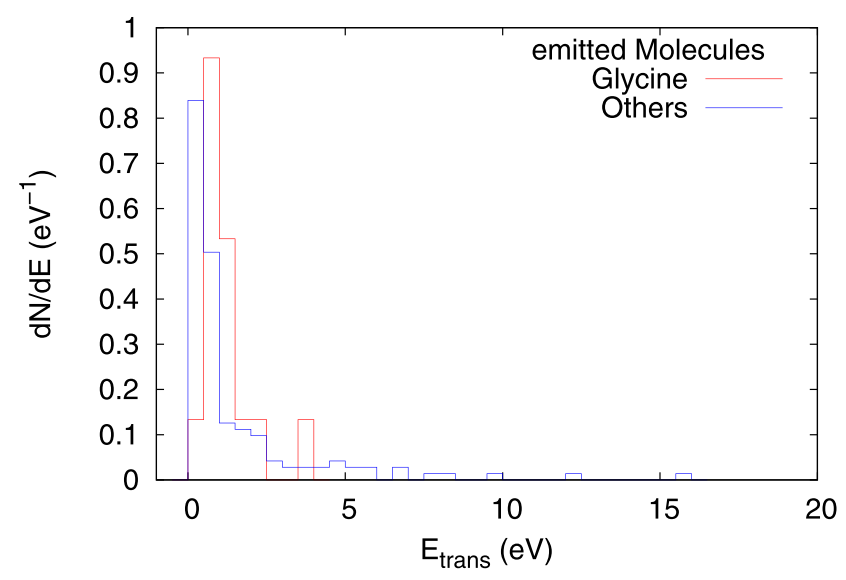

(b)

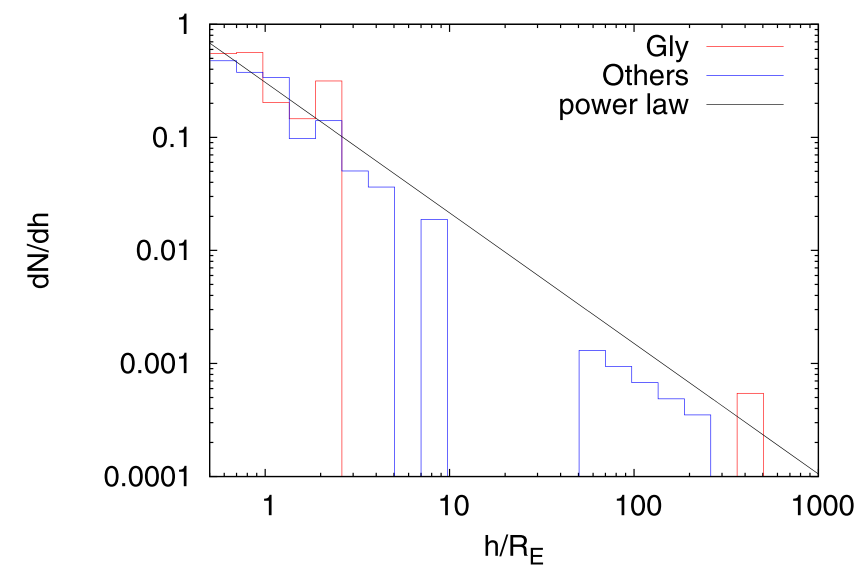

(c)

Figure 8. Distribution of (a) velocity, $v$, and (b) translational energy, $E_{\text {trans }}$, of ejecta. Panel (c) displays the height, $h$, reached by ejecta above Europa's surface in units of Europa's radius, $R_{\mathrm{E}}$. Speeds below the escape velocity from Europa, $v_{\text {escape }}=2.03 \mathrm{~km} \mathrm{~s}^{-1}$, are highlighted in (a). The line in (c) gives a dependence $\propto h^{-1.16}$.

forward direction — centered broadly around $\varphi=0$ —and at a polar angle of $\theta \simeq 35^{\circ}$, i.e., roughly at a direction perpendicular to the ion track. These data are thus in agreement with

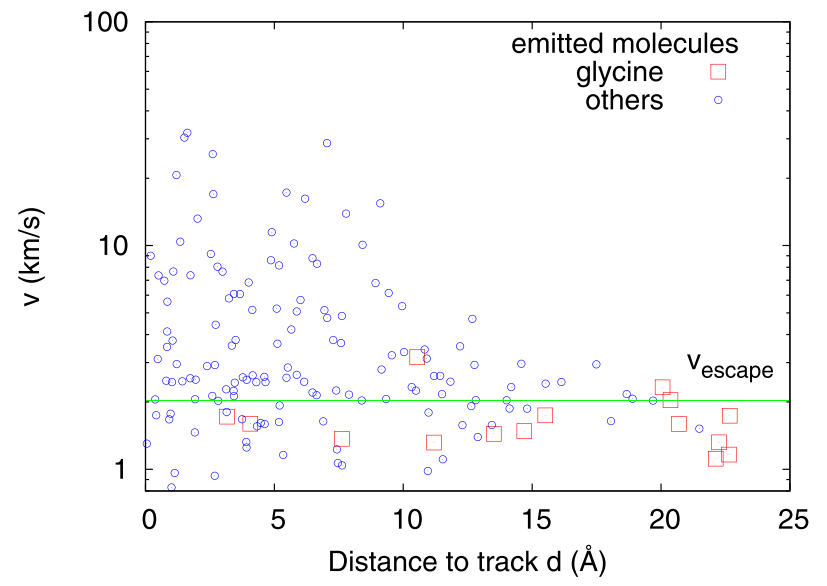

(a)

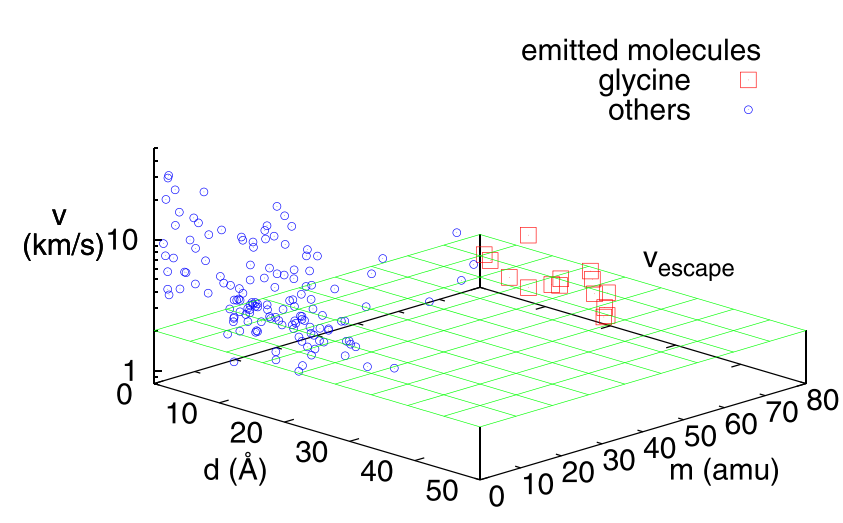

(b)

Figure 9. (a) Velocity of ejecta as a function of the radial distance, $d$, of their original location to the ion track. Panel (b) shows the same data resolved with respect to the ejecta mass, $m$. The escape velocity from Europa, $v_{\text {escape }}=2.03 \mathrm{~km} \mathrm{~s}^{-1}$, is highlighted.

the idea that the high pressure inside the track gives a momentum to each molecule in the radial direction away from the track axis, which may lead to molecule emission (Ens et al. 1989; Fenyö et al. 1990; Reimann 1995). This so-called "pressure-pulse model" of molecule emission (Johnson et al. 1989) has explained some irradiation experiments for biomolecules (Reimann 1995) and also for polymers (Papaléo et al. 2015). It assumes a sharp impulsive transfer, consistent with our simulations, but the pressure pulse resulting from this ion track might be difficult to describe with equilibrium shock equations, as has been proposed for biomolecule damage near tracks (Toulemonde et al. 2009). Also our finding that intact molecules are ejected from an annular region surrounding the ion impact point-see Figure 7 above-agrees with the predictions of the pressure-pulse model (Fenyö et al. 1990). However, as noted in the discussion of Figure 6, emission from deep sites inside the material is also possible; this will occur by evaporation rather than by pressure-pulse emission and will contribute a diffuse (isotropic) background to the angular emission.

The angular emission pattern of "other" molecules-mainly water - has a more diffuse polar-angle characteristic; its azimuthal 


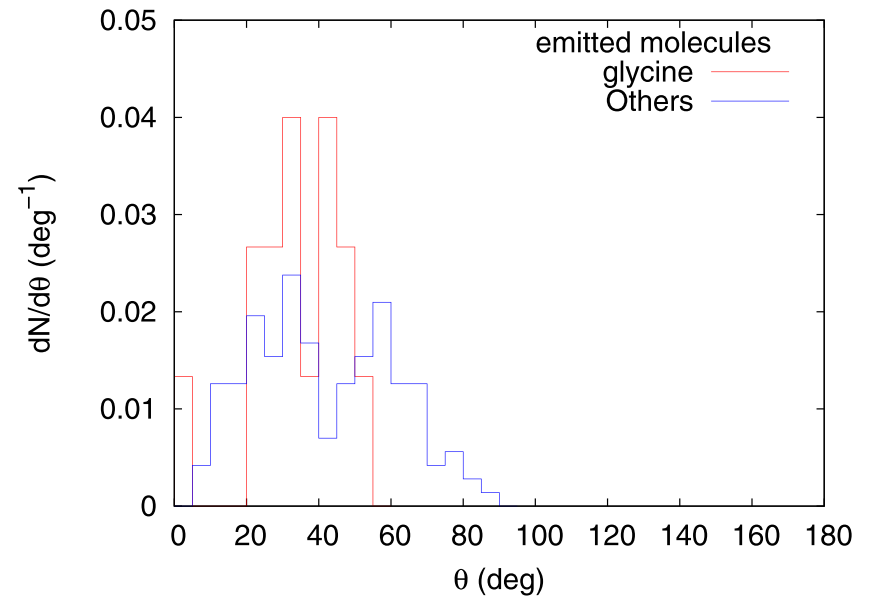

(a)

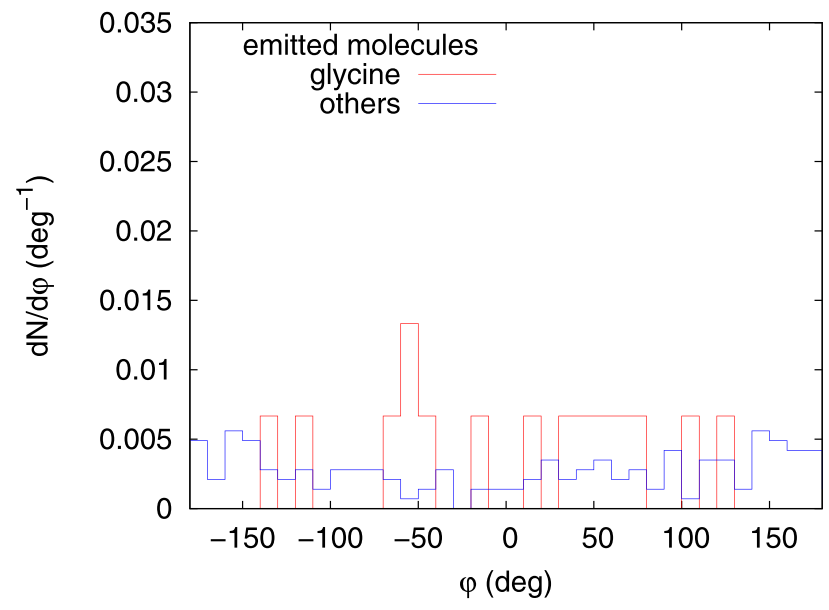

(b)

Figure 10. Distribution of (a) polar angle $\theta$ toward surface normal and (b) azimuthal angle $\varphi$ of emitted molecules.

distribution even appears to slightly favor backwards emission $\left(\phi \simeq 180^{\circ}\right)$. This feature is compatible with water molecules evaporating out of the cylindrical track region, peaking along the track axis. We conclude that while the near-surface glycine emission is compatible with the pressure-pulse scenario, the ejection of molecules from larger depths may operate differently.

\section{Conclusions}

Organic molecules may be present at the surfaces of icecovered moons or of comets. They may be dissociated and chemically transformed by ion irradiation; this irradiation may also eject the molecules into space, thus - at low velocitiesrelocating them, and at higher velocities permanently removing them from their parent body. We used a molecular dynamics simulation to study such processes for the prototypical example of glycine adsorbed at the surface of amorphous water ice. Rather than studying the effect on an isolated molecule, we covered the water ice surface with a glycine layer. As an irradiating ion, we used a $2 \mathrm{MeV}$ sulfur ion, as is typical of the magnetosphere of Jupiter. We obtain the following findings.

1. Glycine shows an abundant spectrum of fragment species formed; among them cyanide $\mathrm{CN}^{-}$, carbon monoxide $\mathrm{CO}$, cyanate $\mathrm{OCN}^{-}$, and carbon dioxide $\mathrm{CO}_{2}$ are most prominent. These findings are in agreement with the experimental work of Portugal et al. (2014) who studied the radiolysis of a pure glycine target under $46 \mathrm{MeV} \mathrm{Ni}$ ion impact.

2. Dissociation of water molecules generates $\mathrm{H}^{+}, \mathrm{OH}^{-}$, and $\mathrm{H}_{3} \mathrm{O}^{+}$. Radical reactions produce $\mathrm{H}_{2}, \mathrm{O}_{2}$, and some $\mathrm{H}_{2} \mathrm{O}_{2}$. Again, this is in agreement with experimental studies of water hydrolysis (Bar-Nun et al. 1985; Baragiola et al. 2005). Due to the presence of $\mathrm{C}$ and $\mathrm{N}$ from glycine fragments, also $\mathrm{CO}_{2}$ and $\mathrm{NH}_{3}$ are generated.

3. Particles may be ejected from the ion track into the vacuum above it. In the case studied here, a total mass of 4379 amu was ejected. For lighter fragments, we find ejection velocities that may by far exceed the escape velocity of Europa. Most ejected glycine molecules $(80 \%)$, however, do not reach the escape velocity.

4. The ejection data (emission angle and velocity) allow to calculate the Kepler orbits of the ejecta around Europa. Our data show that the distribution of heights reached by ejecta above Europa's surface follows a power-law distribution, Equation (1), with an exponent around 1.2. Similarly, the data could be used to obtain the density distribution of a ballistic exosphere created by sputtering.

5. We find that a considerable number of glycines (15) are ejected intact, or with one proton added or subtracted $(\mathrm{Gly} \pm \mathrm{H})$. This is a substantial number in view of the high temperatures in the ion track that might easily fragment glycine molecules. The amount of glycine ejected corresponds to a desorption cross section of around $20 \mathrm{~nm}^{2}$.

6. In contrast to simulations of shocks running through a glycine target (Martins et al. 2013; Koziol \& Goldman 2015), we found no formation of peptide bonds, i.e., $\mathrm{Gly}_{2}$ was not formed. This is understandable as the necessary pressures and temperatures to overcome the energy barrier of $0.9 \mathrm{eV}$ (Kroonblawd et al. 2018; Lindsey et al. 2019) are generated only for small times $(<5 \mathrm{ps})$ in the ion-induced track.

7. For the case of oblique ion incidence studied here, ejecta leave the surface mainly in the forward direction, at a perpendicular direction to the track. This feature is reminiscent of the pressure-pulse model of molecule emission (Reimann 1995).

8. Ejected species may leave the gravitational field of Europa or contribute to its atmosphere, depending on their velocity. This is in line with the observation that Europa holds a tenuous $\mathrm{O}_{2}$ atmosphere, which is believed to be due to sputtering from Europa (Hall et al. 1995; Shematovich \& Johnson 2001; Johnson et al. 2009).

9. Our results demonstrate that intact emission of organic molecules from Europa's surface by ion irradiation is possible. Besides magnetospheric ion impact, artificial irradiation during space missions may also induce ejection (Johnson \& Sundqvist 1992; Johnson et al. 1998; Johnson \& Sundqvist 2018). Since the emission velocities of heavy ejecta are sufficiently small, ejected organic molecules will populate the exosphere surrounding Europa, and might be detectable there.

We acknowledge financial support by the Deutsche Forschungsgemeinschaft within project Ur 32/27-2. Simulations 
were performed at the High Performance Cluster Elwetritsch (RHRK, TU Kaiserslautern, Germany).

\section{ORCID iDs}

Herbert M. Urbassek (1) https://orcid.org/0000-0002$7739-4453$

\section{References}

Altwegg, K., Balsiger, H., Bar-Nun, A., et al. 2016, SciA, 2, e1600285

Anders, C., \& Urbassek, H. M. 2013, NIMPB, 303, 200

Anders, C., \& Urbassek, H. M. 2017, Icar, 282, 351

Anders, C., \& Urbassek, H. M. 2019a, MNRAS, 482, 2374

Anders, C., \& Urbassek, H. M. 2019b, A\&A, 625, A140

Balaji, V., David, D. E., Tian, R., Michl, J., \& Urbassek, H. M. 1995, JPhCh, 99, 15565

Baragiola, R. A., Loeffler, M. J., Raut, U., Vidal, R. A., \& Wilson, C. D. 2005 RaPC, 72, 187

Bar-Nun, A., Herman, G., Rappaport, M. L., \& Mekler, Y. 1985, in Ices in the Solar System, ed. J. Klinger et al. (Dordrecht: Reidel), 287

Berendsen, H. J. C., Postma, J. P. M., van Gunsteren, W. F., DiNola, A., \& Haak, J. R. 1984, JChPh, 81, 3684

Carlson, R. W., Calvin, W. M., Dalton, J. B., et al. 2009, in Europa, ed. R. T. Pappalardo, W. B. McKinnon, \& K. K. Khurana (Tucson, AZ: Univ. Arizona Press), 283

Cassidy, T. A., Paranicas, C. P., Shirley, J. H., et al. 2013, Phys. Stat. Sol., 77, 64

Ens, W., Sundqvist, B. U. R., Håkansson, P., Hedin, A., \& Jonsson, G. 1989 , PhRvB, 39, 763

Fenyö, D., Sundqvist, B. U. R., Karlsson, B. R., \& Johnson, R. E. 1990, PhRvB, 42, 1895

Goldman, N., \& Tamblyn, I. 2013, JPCA, 117, 5124

Grasset, O., Dougherty, M. K., Coustenis, A., et al. 2013, P\&SS, 78, 1

Gudipati, M. S., \& Castillo-Rogez, J. (ed.) 2013, The Science of Solar System Ices (New York: Springer)

Hall, D. T., Strobel, D. F., Feldman, P. D., McGrath, M. A., \& Weaver, H. A. 1995, Natur, 373, 677

Hedin, A., Håkansson, P., Salehpour, M., \& Sundqvist, B. U. R. 1987, PhRvB, 35,7377

Humphrey, W., Dalke, A., \& Schulten, K. 1996, J. Mol. Graphics, 14, 33

Johnson, R. E. 1990, Energetic Charged-Particle Interactions with Atmospheres and Surfaces (Berlin: Springer)

Johnson, R. E., \& Brown, W. L. 1982, NIMPR, 182, 103

Johnson, R. E., Burger, M. H., Cassidy, T. A., et al. 2009, in Europa, ed. R. T. Pappalardo, W. B. McKinnon, \& K. K. Khurana (Tucson: Univ. Arizona Press), 507

Johnson, R. E., Carlson, R. W., Cooper, J. F., et al. 2004, in Jupiter-The Planet, Satellites and Magnetosphere, ed. F. Bagenal, T. Dowling, \& W. B. McKinnon (Cambridge: Cambridge Univ. Press), 485

Johnson, R. E., Killen, R. M., Waite, J. H., Jr., \& Lewis, W. S. 1998, GeoRL 25,3257
Johnson, R. E., Lanzerotti, L. J., \& Brown, W. L. 1982, NIMPR, 198, 147

Johnson, R. E., \& Sundqvist, B. U. R. 1992, PhT, 45, 28

Johnson, R. E., \& Sundqvist, B. U. R. 2018, Icar, 309, 338

Johnson, R. E., Sundqvist, B. U. R., Hedin, A., \& Fenyö, D. 1989, PhRvB, 40, 49

Kalson, N.-H., Furman, D., \& Zeiri, Y. 2017, ACS Central Science, 3, 1041

Koziol, L., \& Goldman, N. 2015, ApJ, 803, 91

Kroonblawd, M. P., Pietrucci, F., Saitta, A. M., \& Goldman, N. 2018, Journal of Chemical Theory and Computation, 14, 2207

Lindsey, R. K., Kroonblawd, M. P., Fried, L. E., \& Goldman, N. 2019, in Computational Approaches for Chemistry Under Extreme Conditions, ed. N. Goldman (Cham: Springer International Publishing), 71

Mainitz, M., Anders, C., \& Urbassek, H. M. 2016, A\&A, 592, A35

Mainitz, M., Anders, C., \& Urbassek, H. M. 2017, NIMPB, 393, 34

Martínez, L., Andrade, R., Birgin, E. G., \& Martínez, J. M. 2009, JCoCh, 30,2157

Martins, Z., Price, M. C., Goldman, N., Sephton, M. A., \& Burchell, M. J. 2013, NatGe, 6, 1045

Meyer, S., Tulej, M., \& Wurz, P. 2017, GI, 6, 1

Monti, S., Corozzi, A., Fristrup, P., et al. 2013, PCCP, 15, 15062

Pakarinen, O. H., Djurabekova, F., Nordlund, K., Kluth, P., \& Ridgway, M. C. 2009, NIMPB, 267, 1456

Papaléo, R. M., Thomaz, R., Gutierres, L. I., et al. 2015, PhRvL, 114, 118302

Pappalardo, R. T., McKinnon, W. B., \& Khurana, K. K. (ed.) 2009, Europa (Tucson, AZ: Univ. Arizona Press)

Paranicas, C., Cooper, J. F., Garrett, H. B., Johnson, R. E., \& Sturner, S. J. 2009, in Europa, ed. R. T. Pappalardo, W. B. McKinnon, \& K. K. Khurana (Tucson: Univ. Arizona Press), 529

Plimpton, S. 1995, JCoPh, 117, 1

Portugal, W., Pilling, S., Boduch, P., Rothard, H., \& Andrade, D. P. P. 2014, MNRAS, 441, 3209

Rahaman, O., van Duin, A. C. T., Goddard, W. A., \& Doren, D. J. 2011, JPCB, 115,249

Reimann, C. T. 1995, NIMPB, 95, 181

Senftle, T. P., Hong, S., Islam, M. M., et al. 2016, npjCM, 2, 15011

Shematovich, V. I., \& Johnson, R. E. 2001, AdSpR, 27, 1881

Tachibana, S., Kouchi, A., Hama, T., et al. 2017, SciA, 3, eaao2538

Teolis, B. D., Shi, J., \& Baragiola, R. A. 2009, JChPh, 130, 134704

Teolis, B. D., Vidal, R. A., Shi, J., \& Baragiola, R. A. 2005, PhRvB, 72, 245422

Teolis, B. D., Wyrick, D. Y., Bouquet, A., Magee, B. A., \& Waite, J. H. 2017, Icar, 284, 18

Toulemonde, M., Surdutovich, E., \& Solov'yov, A. V. 2009, PhRvE, 80, 031913

Urbassek, H. M., Kafemann, H., \& Johnson, R. E. 1994, PhRvB, 49, 786

van Duin, A. C. T., Dasgupta, S., Lorant, F., \& Goddard, W. A., III 2001, JPCA, 105, 9396

Vollhardt, K. P. C., \& Schore, N. E. 2014, Organic Chemistry: Structure and Function (6th ed.; San Francisco: Freeman)

Vorburger, A., \& Wurz, P. 2018, Icar, 311, 135

Wu, Y.-T., \& Adnan, A. 2017, NatSR, 7, 5323

Ziegler, J. F. 2000, SRIM, http://www.srim.org/

Ziegler, J. F., Biersack, J. P., \& Littmark, U. 1985, The Stopping and Range of Ions in Solids (New York: Pergamon) 